\title{
Designing Circular CMUT Cells Using CMUT Biasing Chart
}

\author{
H. Köymen, Senior Member, IEEE, Abdullah Atalar, Fellow, IEEE, \\ and $\mathrm{H}$. Kağan Oğuz, Student Member IEEE
}

\author{
Bilkent University, Electrical and Electronics Engineering Department, Ankara, Turkey \\ e-mail: koymen@ee.bilkent.edu.tr
}

\begin{abstract}
A very accurate equivalent circuit model for circular CMUT cell is recently proposed. As a consequence of this model, the operating characteristics of the cell is derived and presented as a biasing chart for CMUTs. We discuss these operating characteristics and show how the coupling coefficient can be derived from the model. We present an analysis of receiver performance of a CMUT cell. We also demonstrate how to design cells for fixed bias voltage but for different static depression.
\end{abstract}

\section{INTRODUCTION}

We presented a lumped element equivalent circuit for circular CMUT cells in [1]. We showed that this equivalent circuit models the CMUT cell at Finite Element Analysis (FEA) level accuracy. We also presented a chart for operating characteristics, which is derived from the static force equilibrium on the plate. We call this chart CMUT Biasing Chart (CBC).

CMUT has an inherent potential to be designed to have a prespecified transduction properties. CBC describes the operational characteristics of CMUT and provides this option to the designer.

When an external static pressure is present, the static force equilibrium equation can be put into the normalized form given in (1),

$$
\frac{V_{D C}}{V_{r}}=\sqrt{\frac{3\left(X_{P} / t_{g e}-F_{b} / F_{g}\right)}{2 g^{\prime}\left(X_{P} / t_{g e}\right)}}
$$

with

$g^{\prime}(u)=\frac{1}{2 u}\left(\frac{1}{1-u}-\frac{\tanh ^{-1}(\sqrt{u})}{\sqrt{u}}\right)$

where $V_{D C}$ is the bias voltage, $V_{r}$ is the collapse (pull-in) voltage in vacuum for a CMUT with full electrode, $X_{P}$ is the displacement at the center of the plate, $F_{b}$ is the external static force, $t_{g e}$ is the effective gap height, $t_{g}+t_{i} / \varepsilon_{r}$, given in Fig. 1, and $F_{g}$ is a uniformly distributed force required to depress the plate such that $X_{P}=t_{g e} . F_{g}$ is not a physical force acting on CMUT, but it is defined for normalization purposes only.

$\mathrm{CBC}$ is a mapping of the variation of normalized bias voltage with respect to normalized center displacement, $X_{P} / t_{g e}$, of the plate while the mechanical equilibrium is maintained. All variables, the applied voltage, the active force and the displacement, are normalized to appropriate intrinsic values in $\mathrm{CBC}$ and therefore it is a general operating characteristic curve for CMUTs with circular membranes. CBC is plotted in Fig. 2 for different values of normalized static force, $F_{b} / F_{g}$. For example, if we consider a CMUT in vacuum $\left(F_{b} / F_{g}=0\right)$, $X_{P} / t_{g e}$ increases from zero as $V_{D C}$ is increased. A maximum is reached at $V_{D C}=V_{r}$ and at $X_{P} \approx 0.46 t_{g e}$, above which the collapse occurs. In order to maintain the equilibrium after this point, we must decrease $V_{D C}$. However, the equilibrium is unstable for $\mathrm{X}_{\mathrm{P}} / \mathrm{t}_{\mathrm{ge}}>0.46$.

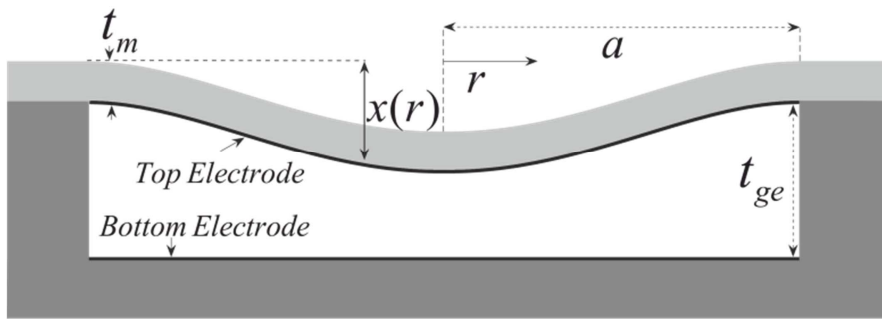

Fig. 1 CMUT geometry

$V_{r}$ is obtained from the static equilibrium equation as,

$V_{r}=8 \frac{t_{m}}{a^{2}} t_{g e}^{3 / 2} t_{m}^{1 / 2} \sqrt{\frac{Y_{0}}{27 \varepsilon_{0}\left(1-\sigma^{2}\right)}}$

which is the same as the voltage reported in [2]. When an external static pressure, $P_{0}$, is present, there is also a static depressing force $F_{b}$ acting on the plate. $F_{b} / F_{g}$ is a measure of relative effect of this force compared to the restoring force due to the compliance of the plate. The plate is pre-depressed by 
this force to $X_{P} / t_{g e}=F_{b} / F_{g}$ before any voltage is applied. When we increase $V_{D C}$, we observe a behavior similar to that in vacuum. For example, the collapse occurs at $X_{P} / t_{g e}=0.74$ when $F_{b} / F_{g}=0.5$, but at a lower $V_{D C} / V_{r}$ of 0.48 . The variation of normalized displacement at collapse and normalized collapse voltage versus $F_{b} / F_{g}$ are all given in [1], (23) and (24) respectively, and plotted in Fig. 2 as the "collapse line".

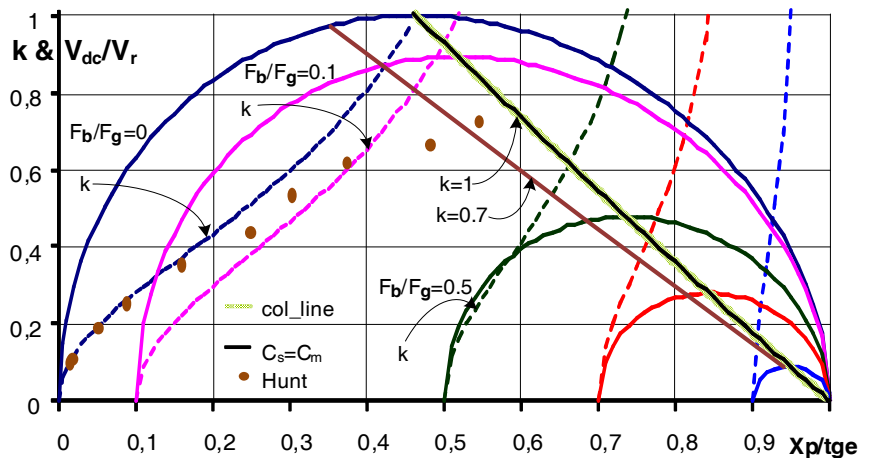

Fig. $2 \mathrm{CBC}$ and coupling coefficient as a function of $X_{P} / t_{g e}$. $V_{D C} / V_{r}$ : solid lines; $k$ : dashed lines

The collapse voltage is the limiting voltage for any bias in the uncollapsed mode of operation. CBC reveals that as the membrane is allowed to be depressed by static environmental pressure, the collapse voltage becomes a smaller fraction of the collapse voltage in vacuum. For example, it is only $0.09 V_{r}$ at $F_{b} / F_{g}=0.9$.

It is possible to show that the quality factor of a cell in air is roughly proportional to the square of the thickness-to-radius ratio, $t_{m} / a$. It is desirable to have this ratio as small as possible for a wider bandwidth. On the other hand, smaller this ratio is, more compliant is the plate and it is depressed more by the static pressure.

CBC allows us to include the static pressure into the design as a requirement. In [3], an airborne CMUT design is described for somewhat different driving conditions, where the required plate swing for maximum radiated power is determined first. Then, CMUT is designed such that $F_{b} / F_{g}$ is set to largest possible level which enables this swing level at the minimum drive voltage amplitude.

\section{COUPLing COEFFICIENT}

\section{A. Coupling coefficient from equivalent circuit}

The small signal model presented in [1] is given in Fig. 3.

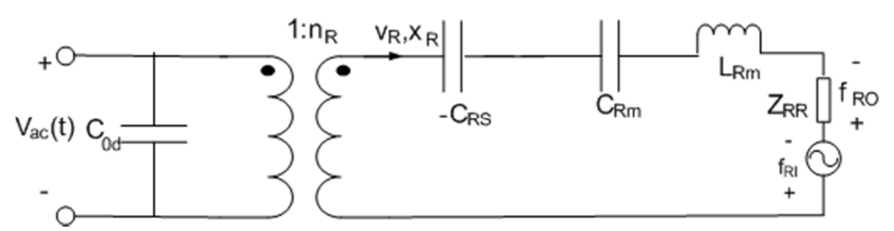

Fig. 3 Small signal equivalent circuit for the rms model A common definition for the electromechanical coupling coefficient is the square root of ratio of converted energy to the total stored energy,

$k \equiv \sqrt{\frac{W_{c}}{W_{t o t}}}$

If we consider only the dynamic energies involved in the transduction, the total and converted energies are,

$W_{t o t_{-} d y n}=\frac{1}{2} V^{2}\left[C_{0 d}+n_{R}^{2}\left(\frac{1}{-C_{R S}}+\frac{1}{C_{R m}}\right)^{-1}\right]$

and

$W_{c_{-} d y n}=\frac{1}{2} V^{2} n_{R}^{2}\left(\frac{1}{-C_{R S}}+\frac{1}{C_{R m}}\right)^{-1}$

respectively. Hence the coupling coefficient becomes:

$k=\frac{1}{\sqrt{1+\frac{C_{0 d}}{n_{R}^{2}} \frac{C_{R S}-C_{R m}}{C_{R m} C_{R S}}}}$

In the presence of a parasitic capacitance, $C_{p}$, at the electrical port, $k$ becomes

$k=\frac{1}{\sqrt{1+\frac{C_{0 d}+C_{p}}{n_{R}^{2}} \frac{C_{R S}-C_{R m}}{C_{R m} C_{R S}}}}$

The coupling coefficient is calculated when the membrane is in a mechanical equilibrium and plotted in Fig. 2 as a function of normalized peak displacement, together with CBC. Note that the coupling coefficient becomes unity $(k=1)$ at the collapse threshold regardless of static depression and the value of the parasitic capacitance.

The collapse threshold and the unity coupling coefficient locus for all static depression conditions are also plotted as collapse line and $C_{R S}=C_{R m}$ lines in the same figure. These two conditions are the same.

It is possible to calculate the bias level at a particular static pressure, required for a specific coupling coefficient value. Bias voltage levels required for coupling coefficient of 0.7 at a particular static external pressure is also depicted in Fig. 2. Note that $k=1$ line is the collapse line. The bias level necessary for $k=0.7$ (the coupling coefficient level of PZT-5), is $0.97 V_{r}$ in vacuum. Although the displacement at this bias level has some clearance from collapse threshold, this voltage 
level is very close to collapse voltage. Hence there is potential for operating CMUT at a high coupling coefficient bias but there is a problem of keeping the CMUT safely in the stable region. Bias level for $k=0.7$ becomes 0.984 of collapsing voltage at $F_{b} / F_{g}=0.9$ (collapsing voltage is $0.0896 V_{r}$ ), which is even closer to the collapse threshold.

The coupling coefficient in vacuum can be determined from CBC as 0.52 when the CMUT is biased at the commonly used level of $V_{D C} / V_{r}=0.9$. The center displacement becomes $X_{P} / t_{g e}$ $=0.26$ at this operating point. When there is static pressure depression of $F_{b} / F_{g}=0.9$, coupling coefficient becomes 0.47 at $V_{D C} / V_{r}=0.9$.

\section{B. Comparison with Hunt's Measurements}

The electrostatic transducer that Hunt studied in Chapter 6 of [4] is similar to a circular CMUT cell in structure, but made of different materials. Hunt modeled this transducer as a parallelplate condenser, and made very accurate measurements to experimentally determine various transducer parameters including coupling coefficient. Hunt defined the depressed capacitance as

$$
C_{0 H}=\frac{C_{0}}{1+x_{0} / t_{g e}}
$$

where $x_{0}$ is the effective displacement representing the displacement in parallel-plate condenser. Experimental determination of $x_{0}$ at different bias levels is done indirectly by measuring $C_{O H}$, and $x_{0}$ is calculated as

$$
1+x_{0} / t_{g e}=\frac{C_{0}}{C_{0 H}}
$$

Measurements show that the collapse occurs when $x_{0} / t_{g e}$ is -0.22 . Hunt found this level premature because the theoretical limit of expected displacement at collapse is $t_{g e} / 3$ in parallel plate condenser. He suspected that the measurements or the curvature of the membrane may be causing this result.

The transducer that Hunt considered had perforations on the rigid electrode and air can move in and out of the gap. Static operation of this transducer corresponds to operation in vacuum for the model in [1]. Using this model, (10) can be written as

$$
1+x_{0} / t_{g e}=\frac{C_{0}}{C_{0 H}}=\frac{C_{0}}{C_{0} g\left(X_{P} / t_{g e}\right)}
$$

Since the collapse occurs at $X_{P} / t_{g e} \approx 0.46$ in vacuum, (11) predicts $x_{0} / t_{g e} \approx-0.18$ instead of $-1 / 3$. Hunt, however, ended up with -0.22 instead of -0.18 for $x_{0} / t_{g e}$ at collapse threshold. The behavior of CMUT depends strongly on its electrical termination as it is discussed in the following section. The termination is not known for these measurements since the method used for measuring the capacitance is not detailed in [4]. If the measurement set up incorporates very high impedance connections for the source, the performance of CMUT is affected. It is assumed that the source impedance of the measurement set up is low enough so that voltage drive conditions prevail. Also, the way $C_{O H}$ is measured in the experiment introduces an effect of mechanical section and results in a larger value for $C_{O H}$. This effect increases as the biasing voltage is increased.

Measurement results are reported in Fig. 6.7 of [4] for coupling coefficient and $x_{0} / t_{g e}$. This data is converted for $X_{P} / t_{g e}$ (within reading accuracy) and plotted on Fig. 2. Measured $k$ values follow model prediction for $k$ and $X_{P} / t_{g e}$ less than 0.4 and 0.2 , respectively. For larger values measurements become erroneous as discussed above.

\section{RECEIVER OPERATION}

The open circuit receiving voltage (OCRV) at very low frequencies (compared to resonance frequency) can also be readily obtained as the transfer function of an open circuit CMUT from Fig. 3 as

$$
\frac{V_{o c}}{\pi a^{2} p}=\frac{1}{n_{R}} \frac{1}{1+\frac{C_{p}+C_{0 d}}{n_{R}^{2}}\left(\frac{1}{C_{R m}}-\frac{1}{C_{R S}}\right)}
$$

where $p$ is incident pressure and $C_{p}$ is the parasitic capacitance. (12) can be written as

$$
\frac{V_{o c}}{p}=\left[\frac{3}{8} \sqrt{\frac{2}{5}} \sqrt{\frac{1-\sigma^{2}}{\varepsilon_{0} Y_{0}}}\left(\frac{a^{2}}{t_{m}}\right) \sqrt{\frac{t_{g e}}{t_{m}}}\right] h\left(\frac{X_{P}}{t_{g e}}, \frac{F_{b}}{F_{g}}, \frac{C_{p}}{C_{0}}\right)
$$

in $\mathrm{V} / \mathrm{Pa}$, where all dimensions are in meters. (13) implies that the plate diameter must be as large as possible and it must be thin compared to both the diameter and the effective gap for highest sensitivity. When expressed in $\mathrm{dB},(13)$ becomes

$$
\begin{aligned}
20 \log \left(\frac{V_{o c}}{p}\right) & =20 \log \left(\frac{3}{8} \sqrt{\frac{2}{5}} \sqrt{\frac{1-\sigma^{2}}{\varepsilon_{0} Y_{0}}} a\right) \\
+ & 20 \log \left(\frac{a}{t_{m}} \sqrt{\frac{t_{g e}}{t_{m}}}\right)+20 \log \left[h\left(\frac{X_{P}}{t_{g e}}, \frac{F_{b}}{F_{g}}, \frac{C_{p}}{C_{0}}\right)\right]
\end{aligned}
$$

For a CMUT of $1 \mathrm{~cm}$ diameter, the first term is $-46.5 \mathrm{~dB} / /(\mathrm{V} / \mathrm{Pa})$, or $-166.5 \mathrm{~dB} / /(\mathrm{V} / \mu \mathrm{Pa})$ for $\mathrm{Y}_{0} \approx 100 \mathrm{GPa}$. This is a very high sensitivity compared to PZT based hydrophones. Second term depends on cell dimensions and is $10 \mathrm{~dB}$ or higher in typical designs of present practice.

The third term, $\boldsymbol{h}\left(\frac{X_{P}}{t_{g e}}, \frac{F_{b}}{F_{g}}, \frac{C_{p}}{C_{0}}\right)$, is a function of the normalized parasitic capacitance and the operational parameters, the normalized static displacement and force. This term is plotted in Fig. 4 for $C_{p}=0$ and $C_{p}=C_{0}$. It can be clearly seen that critical biasing improves the receiver sensitivity dramatically. However, it must be noticed that the maximum sensitivity 
occurs at a static displacement above the collapse threshold for voltage drive operation, when the parasitic capacitance is negligible. For example, in vacuum the collapse occurs at a normalized displacement of about 0.63 compared to 0.46 . The clamp capacitance is not shunted under open circuit operating conditions, and the collapse occurs when

$$
\frac{1}{C_{R S}}=\frac{1}{C_{R m}}+\frac{n_{R}^{2}}{C_{p}+C_{0 d}}
$$

instead of $C_{R S}=C_{R m}$, When $C_{p}$ is significant, the static displacement required for collapse decreases. If there is excessive parasitic capacitance such that $C_{p} \gg C_{0}$, however, collapse displacement approaches to that of voltage drive condition.

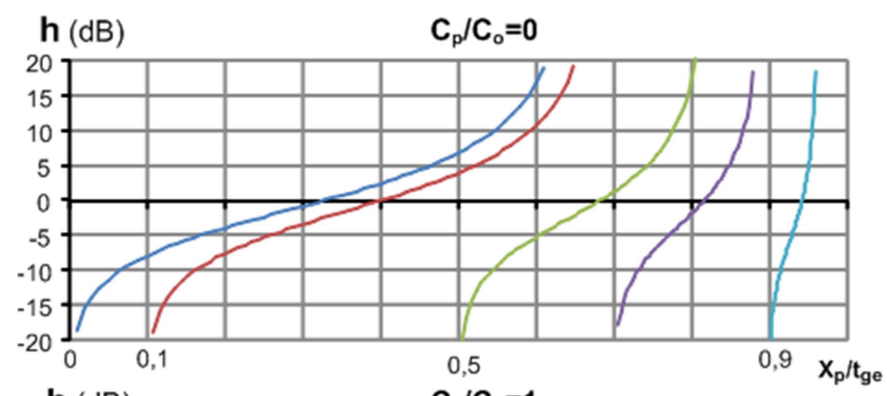

$$
\text { h (dB) }
$$

$\mathrm{C}_{\mathrm{p}} / \mathrm{C}_{\mathrm{o}}=1$

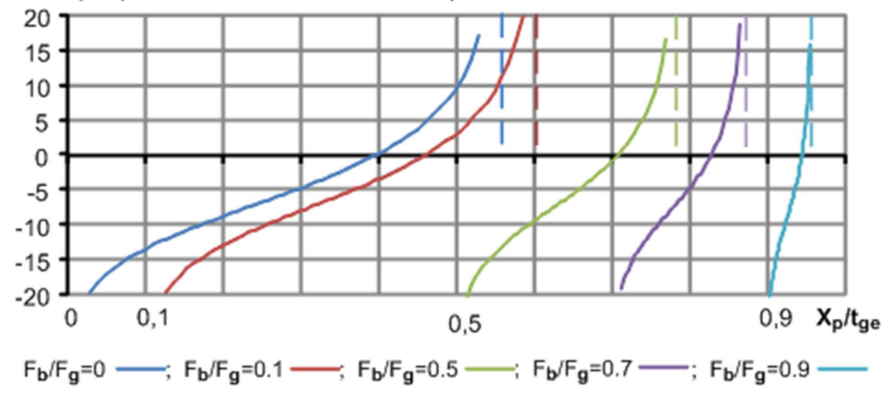

Fig. 4 Receiver sensitivity multiplier $\boldsymbol{h}\left(\frac{X_{P}}{t_{g e}}, \frac{F_{b}}{F_{g}}, \frac{C_{p}}{C_{0}}\right)$

\section{CMUT DIMENSIONS}

The mechanical resonance frequency is

$$
\omega_{m}^{2}=\frac{1}{C_{R m} L_{R m}}
$$

in terms of equivalent circuit parameters. Using (3) and (16) with

$$
\frac{F_{b}}{F_{g}}=\frac{\pi a^{2} P_{0} / 3}{t_{g e} / 5 C_{R m}}
$$

$$
\begin{aligned}
& t_{g e}=V_{r} \sqrt{\left(\frac{9}{4} \frac{\varepsilon_{0}}{P_{0}}\right)\left(\frac{F_{b}}{F_{g}}\right)} \\
& t_{m}=\frac{1}{t_{g e}}\left(\frac{5}{3} \frac{P_{0}}{\rho}\right)\left(\frac{1}{\omega_{m}^{2}} \frac{1}{F_{b} / F_{g}}\right) \\
& a=\sqrt{\frac{t_{m}}{\omega_{m}}} \sqrt[4]{\frac{80}{9} \frac{Y_{0}}{\left(1-\sigma^{2}\right) \rho}}
\end{aligned}
$$

The bias voltage to be used must be within the available dc voltage level. Consider a plate of material with $Y_{0}$ of $130 \mathrm{GPa}$, Poisson's ratio of 0.27 and density of $3.27 \mathrm{~g} / \mathrm{cm}^{3}$. If we wish to operate the cell at a depression level of $F_{b} / F_{g}=0.1$ and at a collapse voltage of $35 \mathrm{~V}$, we find the required $V_{r}$ as $39.2 \mathrm{~V}$ using (24) in [1]. At 1 atm environmental pressure, $t_{g e}$ is 0.175 micrometer. If the required mechanical resonance frequency is $5 \mathrm{MHz}$, plate thickness, $t_{m}$, becomes 2.95 micrometers and the diameter, $a$, is 42.8 micrometers.

Should we wish to operate the cell at the same collapse voltage $(35 \mathrm{~V})$ but at a higher depression of $F_{b} / F_{g}=0.3, V_{r}$ becomes $51.3 \mathrm{~V}$. Again at the same environmental pressure and for the same mechanical resonance frequency, $t_{g e}, t_{m}$ and $a$ are $0.397,0.434$ and 16.4 micrometers, respectively. Note that when the depression level is increased, $a / t_{m}$ ratio also increases from 14.5 to 37.8 and the cell's bandwidth becomes wider.

The above design exercise can similarly be done for a specific $V_{D C} / V_{c o l}$ ratio for a fixed dc voltage, instead of collapse voltage. Also actual operational resonance frequency can be used instead of $\omega_{m}$, by including other relevant elements in the equivalent circuit into the frequency equation.

\section{ACKNOWLEDGMENT}

This work is supported in part by Turkish Scientific and Research Council (TUBITAK) under project grant 110E216.

\section{REFERENCES}

[1] Köymen, H., Atalar, A, Aydoğdu, E, Kocabaş, C, Oğuz, H.K., Olçum, S, Özgürlük, A., Ünlügedik, A., "An improved lumped element nonlinear circuit model for a circular CMUT cell," IEEE Trans. Ultrason. Ferroelectr. Freq. Control, vol. 59, no. 8, pp. 1791-1799, August 2012.

[2] S. Olcum, M. Senlik, and A. Atalar, "Optimization of the gainbandwidth product of capacitive micromachined ultrasonic transducers," IEEE Trans. Ultrason. Ferroelectr. Freq. Control, vol. 52, no. 12, pp.2211-2219, Dec. 2005.

[3] Ünlügedik, A., Atalar, E, Kocabaş, C, Oğuz, H.K, Köymen, H., "Electrically Unbiased Driven Airborne Capacitive Micromachined Ultrasonic Transducer Design," in 2012 IEEE Ultrasonics Symposium, Dresden, October, 2012.

[4] F.V. Hunt, Electroacoustics, The Analysis of Transduction and its Historical Background, 1954, reprint American Institute of Physics, 1982.

we determine the CMUT dimensions uniquely as 